\title{
Conventional and Advanced Imaging in Neuromyelitis Optica
}

\author{
Y. Barnett, I.J. Sutton, M. Ghadiri, L. Masters, R. Zivadinov, and M.H. Barnett
}

\begin{abstract}
SUMMARY: Myelitis and optic neuritis are prototypic clinical presentations of both multiple sclerosis and neuromyelitis optica. Once considered a subtype of multiple sclerosis, neuromyelitis optica, is now known to have a discrete pathogenesis in which antibodies to the water channel, aquaporin 4, play a critical role. Timely differentiation of neuromyelitis optica from MS is imperative, determining both prognosis and treatment strategy. Early, aggressive immunosuppression is required to prevent the accrual of severe disability in neuromyelitis optica; conversely, MS-specific therapies may exacerbate the disease. The diagnosis of neuromyelitis optica requires the integration of clinical, MR imaging, and laboratory data, but current criteria are insensitive and exclude patients with limited clinical syndromes. Failure to recognize the expanding spectrum of cerebral MR imaging patterns associated with aquaporin 4 antibody seropositivity adds to diagnostic uncertainty in some patients. We present the state of the art in conventional and nonconventional MR imaging in neuromyelitis optica and review the place of neuroimaging in the diagnosis, management, and research of the condition.
\end{abstract}

ABBREVIATIONS: AQP4 = aquaporin 4; LESCL = longitudinally extensive spinal cord lesion; $\mathrm{MT}=$ magnetization transfer; NMO = neuromyelitis optica

N euromyelitis optica (NMO) is a severe inflammatory demyelinating disorder of the CNS that is distinct from multiple sclerosis. Once considered to be a monophasic illness defined by the co-occurrence of acute bilateral visual loss and longitudinally extensive myelitis, ${ }^{1} \mathrm{NMO}$ is now recognized as a predominantly relapsing condition in which these index events may be separated by years and, in some cases, decades. ${ }^{2}$ The past 10 years have seen dramatic advances in the understanding of NMO pathogenesis, heralded by the discovery of the "NMO-Immunoglobulin G" antibody in the serum of approximately $70 \%$ of patients with the condition $^{3}$ and the subsequent identification of the target antigen,

From the Sydney Neuroimaging Analysis Centre (Y.B., M.H.B.), Sydney, Australia; Brain and Mind Research Institute (Y.B., M.G., L.M., M.H.B.), University of Sydney, Sydney, Australia; Department of Medical Imaging and Neurology (Y.B., I.J.S.), St Vincent's Hospital, Sydney, Australia; and Buffalo Neuroimaging Analysis Center (R.Z.), Department of Neurology, University of Buffalo, Buffalo, New York.

Y. Barnett was the recipient of the Biogen Idec Neuroradiology Fellowship at the Brain and Mind Research Institute in 2012. No specific grant support was obtained for this review.

Y. Barnett codrafted the manuscript and assessed all included neuroimaging. I.J. Sutton provided important clinical input and revised the manuscript critically for important intellectual content. L. Masters reviewed the included neuroimaging. M. Ghadiri and R. Zivadinov revised the manuscript critically for important intellectual content. M.H. Barnett conceived and codrafted the manuscript.

Please address correspondence to Michael H. Barnett, MD, Sydney Neuroimaging Analysis Centre, Brain and Mind Research Institute, Lvl 4, 94 Mallett St, Camperdown NSW 2050 Australia; e-mail: michael@snac.com.au

- Indicates open access to non-subscribers at www.ajnr.org

Indicates article with supplemental on-line table.

http://dx.doi.org/10.3174/ajnr.A3592 aquaporin 4 (AQP4), ${ }^{4}$ a cell membrane water channel concentrated on astrocyte foot processes at the glial limiting membrane surrounding blood vessels and in association with the pia mater. In keeping with the notion that NMO is an "autoimmune astrocytopathy" and in contradistinction to MS, biopsy studies of evolving lesions show fragmentation and loss of astrocytes that precede demyelination ${ }^{5}$ and the immunopathologic signature of granulocyte infiltration with perivascular immunoglobulin and complement deposition. ${ }^{6}$

The identification of AQP4 antibodies in patients with only a single index event, in some patients with relapsing optic neuropathy, ${ }^{7}$ and in patients with more recently recognized patterns of cerebral disease ${ }^{8}$ predicts progression to $\mathrm{NMO}^{9-11}$ and has led to the introduction of the term "NMO spectrum disorder. " However, false-positive AQP4 antibody results have been reported in patients with definite MS, and the classification and pathogenesis of clinical and radiologic syndromes consistent with classic relapsing $\mathrm{NMO}$, but without serum AQP4 antibodies, remain uncertain.

Current diagnostic criteria (Table 1) still mandate the occurrence of both optic neuritis and myelitis and at least 2 of 3 supportive criteria, including longitudinally extensive spinal cord lesion (LESCL), seropositivity for immunoglobulin G antibodies that bind AQP4 on astrocytes, and brain MR imaging nondiagnostic for MS. ${ }^{12}$ While the presence of LESCLs is the radiologic signature of NMO, the spectrum of MR imaging changes associated with the condition has been significantly expanded by the 
Table 1: Diagnostic criteria for the diagnosis of NMO

\section{Criteria}

\section{Optic neuritis}

Acute myelitis

At least 2 of the following 3 supportive criteria:

Contiguous spinal cord MRI lesion extending over at least 3

vertebral segments

Onset brain MRI not meeting the diagnostic criteria for MS

NMO-IgG seropositivity status

Note:-lgG indicates immunoglobulin G

recognition of an array of characteristic brain lesion patterns ${ }^{8}$ and the introduction of multiparametric and advanced MR imaging techniques in the assessment of the disease.

Neuroimaging has an increasingly important role in both the diagnosis and management of this potentially devastating condition. First, timely differentiation of NMO from MS is critical and the motivator for this review: Early and aggressive immunosuppression is required to prevent or reduce potentially severe disability in NMO, whereas the application of MS-specific therapies can exacerbate the disease ${ }^{13,14}$ or even precipitate severe atypical relapses. ${ }^{5,15}$ Second, serial imaging has an important role in disease monitoring and informs understanding of the temporal aspects of NMO pathophysiology. Third, routine imaging surveillance is necessary to monitor for complications of immunosuppressive therapy. In this review, we summarize state-of-the-art conventional and nonconventional MR imaging in NMO and its place in the clinical management and research of the disease.

\section{CONVENTIONAL SPINE MR IMAGING \\ Typical Spinal Cord Imaging Features}

The presence of LESCLs ( $\geq 3$ vertebral segments) that preferentially involve spinal central gray matter is characteristic of NMO. ${ }^{12,16}$ LESCLs in NMO are T2 hyperintense and frequently T1 hypointense. They may acutely expand the cord and patchily enhance throughout their length (Fig $1 A-C)$. T1 hypointensity $^{16-18}$ and cord atrophy (Fig $\left.1 D\right)^{19}$ are prominent findings in established NMO, reflecting the severity of tissue injury and a propensity for recurrent bouts of inflammatory activity to occur in old lesions. In such cases, we have observed short segments of gadolinium enhancement within pre-existing T2 LESCLs during acute spinal relapse (Fig $1 A,-B$ ). Additionally, short-segment T2hyperintense lesions are described in $\mathrm{NMO}^{20}$ and should not necessarily deter one from the diagnosis, particularly in the presence of other typical neuroimaging findings. While cord pathology in NMO is traditionally associated with a severe clinical phenotype, including the need for ventilatory support in association with high cervical lesions, excellent clinical and radiologic recovery may be seen in some patients. This observation implicates reversible vasogenic edema, a phenomenon described in cerebral NMO lesions ${ }^{8}$ and attributed to transient functional impairment of water transport mediated by AQP4 antibodies, ${ }^{21,22}$ in the pathogenesis of some NMO cord syndromes.

\section{Differential Diagnosis of the LESCL}

Although a confluence of multiple short-segment MS lesions may occasionally mimic the MR imaging features of spinal NMO, contiguous T2 hyperintensity of central gray matter over many segments is rarely observed in MS. ${ }^{12,16}$ Differentiation of spinal
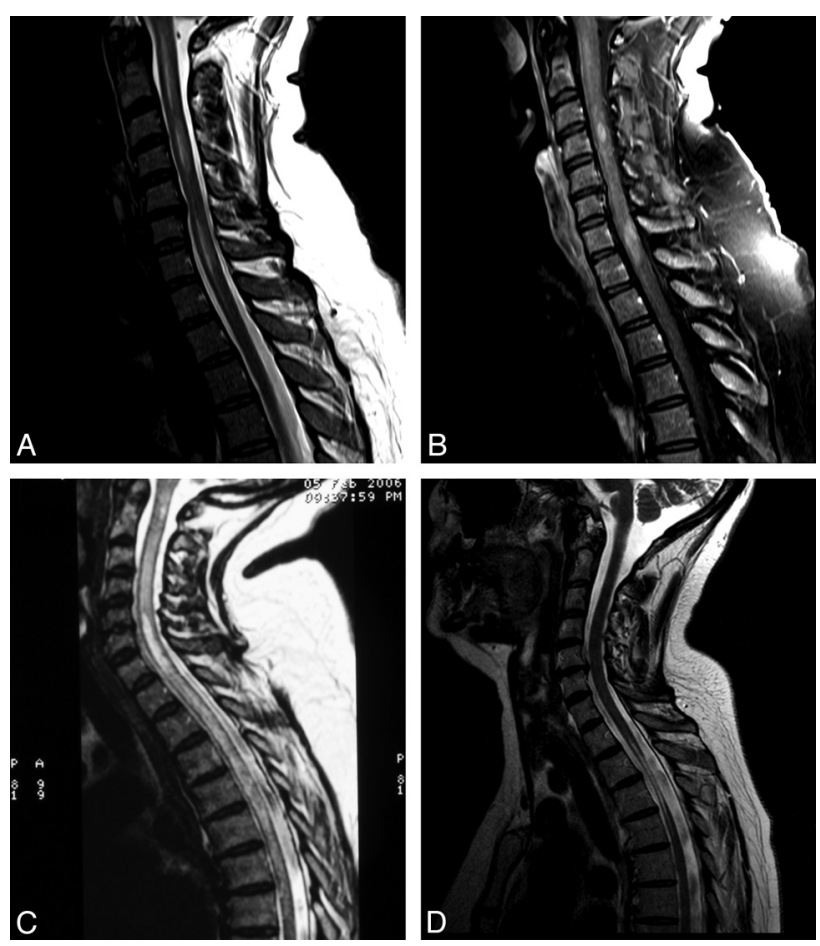

FIG 1. A, T2WI shows extensive patchy pericentral signal change extending from the cervicomedullary junction to the T1 vertebral level in acute symptomatic spinal AQP4-positive NMO. B, Contrast-enhanced TIWI shows that short-segment gadolinium enhancement is present within the cord at the $\mathrm{C} 4$ and $\mathrm{C} 7 / 8$ vertebral levels. C, T2WI shows prototypic longitudinally extensive holocord inflammation in acute symptomatic AQP4-positive NMO relapse. $D, T 2 \mathrm{WI}$ shows severe atrophy in a chronic thoracic spinal cord NMO lesion spanning 3 vertebral segments (T2-T4).

\section{Table 2: Differential diagnosis of LESCLs ${ }^{a}$}

\section{Table 2: Differential diagnosis}

$\mathrm{NMO} / \mathrm{NMO}$ spectrum disorder

Idiopathic isolated or relapsing (AQP4 negative) transverse myelitis Myelitis associated with systemic autoimmunity (Sjögren/systemic

lupus erythematosus)

Postinfectious myelitis

MS: confluent multiple short-segment lesions

Neurosarcoidosis

Behçet disease

Paraneoplastic myelitis

Vascular causes (spinal cord infarction, dural arteriovenous fistula)

${ }^{a}$ Excludes infective/metabolic myelitides and spinal cord tumors.

NMO and other immune-mediated myelitides, such as those associated with Sjögren disease or systemic lupus erythematosus, is more difficult; long cord lesions with a severe clinical phenotype are well-described in the context of systemic autoimmunity (Table 2). ${ }^{23,24}$ However, autoantibodies directed to antigens other than AQP4 are often also detectable in NMO sera, ${ }^{25,26}$ and a proportion of patients have clinical features of systemic autoimmunity. Of 8 patients with Sjögren syndrome-associated myelopathy identified by Kim et al, ${ }^{27} 7$ met the diagnostic criteria for NMO, and AQP4 antibodies were present in $4 / 5$ patients whose serum was available for testing. It is, therefore, likely that many of the Sjögren syndrome-associated myelopathy cases described before the advent of AQP4 antibody testing had coexistent NMO. Other inflammatory disorders that should be considered in patients 

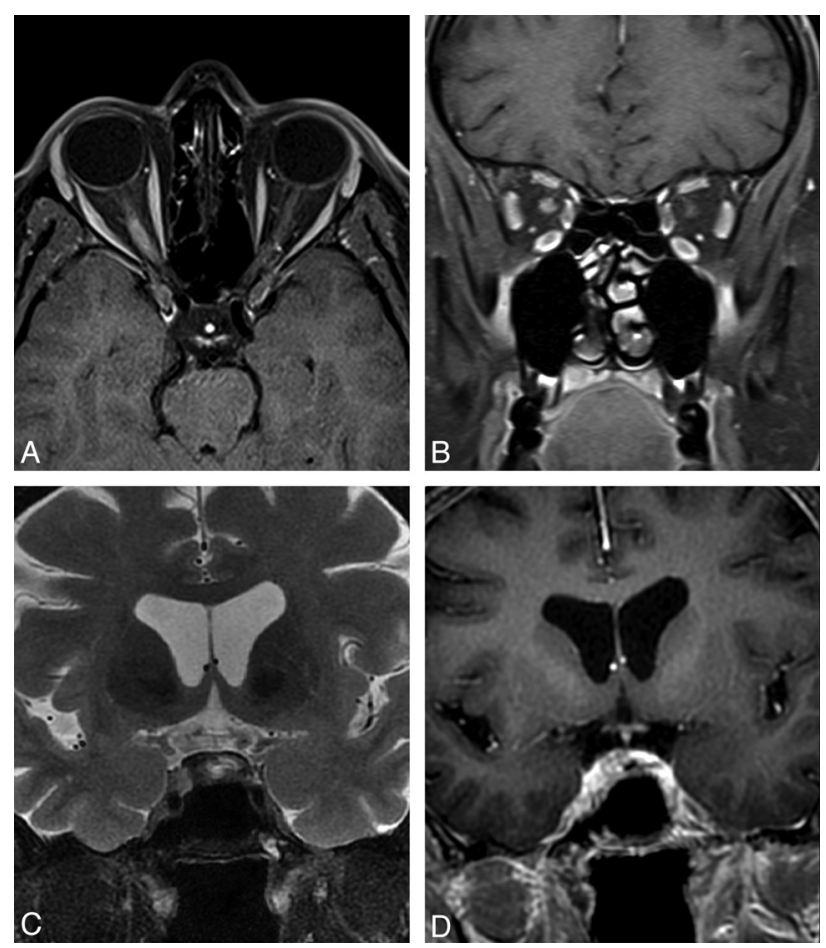

FIG 2. $A$ and $B$, Contrast-enhanced fat-suppressed TIWI shows acute swelling and enhancement of the right optic nerve during symptomatic AQP4-positive NMO relapse with severe right-eye visual loss. C, Fat-suppressed T2WI. D, Contrast-enhanced fat-suppressed TTWI shows chiasmatic hyperintensity and enhancement during symptomatic AQP4-positive NMO relapse with severe visual impairment affecting the entire field of vision in the left eye and the temporal hemifield of the right eye.

with LESCLs include neurosarcoidosis and postinfectious myelitis; while surface enhancement due to meningeal involvement is more common in these conditions, they may be indistinguishable from spinal NMO on radiologic grounds. While rare, paraneoplastic myelitis merits consideration in patients with an isolated LESCL, and testing for serum antibodies to onconeural antigens, including collapsing response mediator protein 5, amphiphysin, or glutamic acid decarboxylase, is advisable in such cases. ${ }^{28}$ Infective and metabolic myelitides are rarely confused with NMO on clinical grounds and are beyond the scope of this review. Of the noninflammatory causes of LESCL, spinal dural arteriovenous fistula is perhaps the most important treatable differential diagnosis.

\section{CONVENTIONAL OPTIC NERVE MR IMAGING}

Long-segment involvement of the optic nerve associated with acute swelling and enhancement (Fig 2A, -B), particularly when bilateral or extending posteriorly into the optic chiasm (Fig $2 C$, $-D$ ), should raise the suspicion of NMO in the appropriate clinical context. $^{29-31}$

\section{CONVENTIONAL BRAIN MR IMAGING}

Cerebral involvement in NMO has traditionally been considered exceptional; indeed, lack of brain involvement was one of the major supportive diagnostic criteria published by Wingerchuk et al in $1999 .^{2}$ However, in 2006, the same group observed brain abnormalities, predominantly asymptomatic, in up to $60 \%$ of a
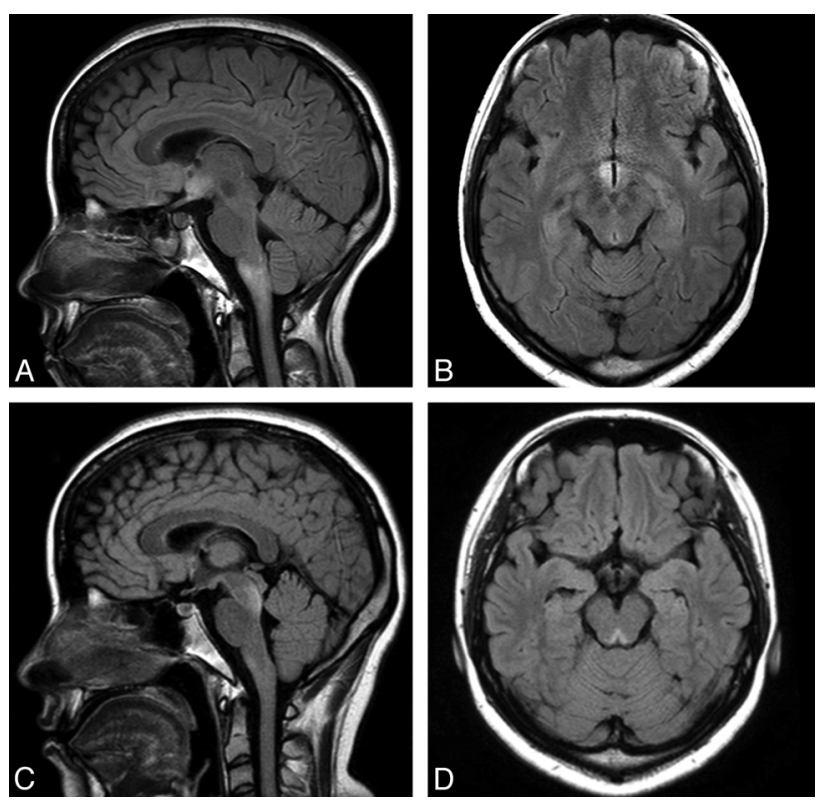

FIG 3. Typical periependymal lesions following the known distribution of AQP4. A and B, FLAIR MR imaging shows hypothalamic and medullary lesions in symptomatic AQP4-positive NMO spectrum disorder presenting with diplopia, dysarthria, dysphagia, and a rightsided sensorimotor syndrome. $C$ and $D$, FLAIR MR imaging shows midbrain tegmentum lesions in the same patient during a second symptomatic attack, presenting with diplopia and clinical evidence of bilateral internuclear ophthalmoplegia. (Images courtesy of Prof. Junichi Kira and Dr. Koji Shinoda, Kyushi University, Kyushi, Japan.)

cohort of patients with clinically defined NMO. ${ }^{32}$ A series of independent reports has subsequently expanded on these findings and defined a spectrum of brain lesion types in up to $93 \%$ of patients with $\mathrm{NMO}$ and $\mathrm{AQP} 4$ antibody-positive NMO spectrum disorder. ${ }^{8,9,19,30,33,34}$ While "typical " NMO lesions (Fig 3) mirror the distribution of AQP4, ${ }^{35,36}$ a number of less specific brain MR imaging changes are increasingly recognized. ${ }^{8}$ Individual brain lesions in NMO have a variable appearance but are often of heterogeneous signal intensity with blurred margins. ${ }^{34}$ The mechanisms and extent of injury also vary from lesion to lesion. Reflecting the now well-described pathology of $\mathrm{NMO},{ }^{5,37}$ extensive cavitation may follow bouts of brain inflammation and result in permanent and severe cognitive dysfunction (Fig $4 E,-F$ ). Conversely, astrocyte destruction is an unlikely pathologic substrate for clinically silent and evanescent hemispheric lesions observed in some patients with $\mathrm{NMO}^{8}$

\section{MR Imaging Lesion Distribution}

Nonspecific punctate or small $(<3 \mathrm{~mm})$ lesions in the deep or, less commonly, subcortical white matter are frequent ${ }^{8,9,32,33}$ and usually asymptomatic. Such lesions may appear MS-like in 10\%$12.5 \%$ of cases, ${ }^{12,19,32,38}$ and in most series, only occasionally fulfill the Barkhof criteria ${ }^{39}$ for the diagnosis of MS in patients who are seropositive for AQP4 antibodies. ${ }^{20}$ To the authors' knowledge, the Magnetic Resonance in Multiple Sclerosis (MAGNIMS) criteria for dissemination in space, which have been incorporated into the most recent iteration of the McDonald criteria for the diagnosis of MS, ${ }^{40}$ have not been formally evaluated in patients with NMO. 

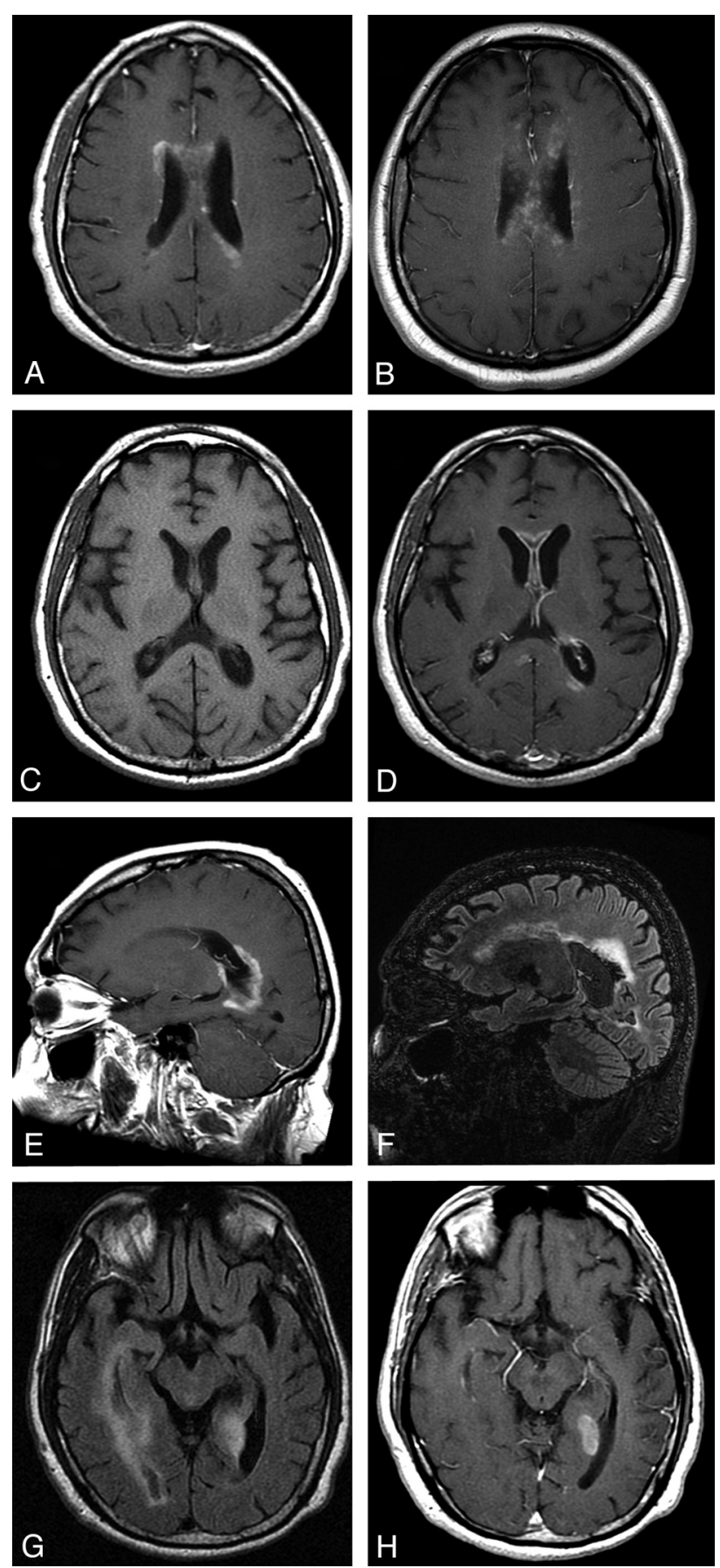

FIG 4. Periependymal/periventricular lesions in acute AQP4-positive NMO. A, Contrast-enhanced TTWI shows ill-defined periventricular cloudlike enhancement. While relatively specific for NMO, similar patterns can be observed in other inflammatory CNS diseases, such as active neurosarcoidosis ( $B$, Contrast-enhanced TIWI). $C$ and $D, T I W I$ and contrast-enhanced TTWI demonstrate pencil-thin ependymal enhancement involving the anterior horns of the lateral ventricles in AQP4-positive NMO. E, Contrast-enhanced TTWI shows thick periependymal enhancement around the posterior horn of the lateral ventricle in acute symptomatic cerebral AQP4-positive NMO relapse. $F, 3 T$ follow-up FLAIR MR imaging shows ventriculomegaly and periventricular encephalomalacia and gliosis. $G$ and $H$, FLAIR MR imaging and contrast-enhanced TIWI shows extensive periependymal disease involving the right lateral ventricle and a discrete, welldemarcated enhancing lesion abutting the posterior horn of the left lateral ventricle.
A number of relatively specific lesion types are helpful in the radiologic diagnosis of NMO:

1) Typical lesions, which follow the distribution of AQP4, are found in the periependymal regions surrounding the third ventricle, cerebral aqueduct, and fourth ventricle (Fig 3). ${ }^{35,36,38,41}$ Lesions within the thalamus and hypothalamus are usually of this type and may be extensive. ${ }^{36,42}$ While often asymptomatic, hypothalamic lesions have been described in patients with NMO with the syndrome of inappropriate antidiuretic hormone secretion ${ }^{43}$; narcolepsy ${ }^{44}$; behavioral change ${ }^{42}$; and hypothermia, hypotension, hypersomnia, and obesity. ${ }^{45}$ Dorsal brain stem lesions adjacent to the fourth ventricle, which may be contiguous with cervical spinal cord lesions, can result in a variety of bulbar symptoms, among which intractable hiccups, nausea, and vomiting are relatively specific for NMO and attributable to involvement of the area postrema and the nucleus tractus solitarius. Additionally, linear medullary lesions have been reported in $48 \%$ of Chinese patients with NMO and discriminated NMO from MS with a high predictive value. $^{46}$

2) Periependymal/periventricular and corpus callosum lesions situated in proximity to the lateral ventricles are common to both MS and NMO. However, the radially oriented, well-demarcated perivenular lesions (Dawson fingers) that characterize MS are absent in most (but not all) cases of NMO; rather, NMO lesions tend to extend along the walls of the lateral ventricles in close association with the ependymal lining (Fig 4). Extension into the white matter of the hemispheres may form large, confluent lesions. Multiple, edematous lesions with heterogeneous signal intensity (higher in the rim and lower in the core of the lesions) in the body of the corpus callosum ("marbled pattern"), ${ }^{47}$ and diffuse involvement and swelling of the splenium may be notable in the acute phase of the disease (Fig $5 A-C$ ). Such lesions may diminish in size and intensity or even disappear on follow-up imaging ${ }^{47}$; however, chronic cystic changes have been described in the genu and splenium of corpus callosum, ${ }^{34}$ and we documented severe atrophy of the callosum at 2.5-year follow-up in 1 case (Fig 5D).

3) Large, confluent, and heterogeneous lesions occur in the cerebral hemispheric white matter in some cases of NMO (Fig $5 E$ ), possibly with a predilection for patients from the Far East and Africa and among children. ${ }^{48}$ These lesions do not exert mass effect ${ }^{9}$ and often vanish following steroid therapy or, in some cases, spontaneously. The pathology of these lesions, which may be contiguous with periependymal lesions or have a "spilled ink" appearance along white matter tracts, ${ }^{8}$ is uncertain, though nondestructive intramyelinic edema has been described in some pathologic studies. ${ }^{21}$ The evanescent nature and presence of facilitated diffusion (Fig $5 F)^{49}$ within lesions also implicates vasogenic edema, potentially due to a reversible or limited astrocytopathy, as the underlying pathology. ${ }^{21,22,50}$ Hemispheric lesions may be clinically silent or result in focal neurologic deficits.

4) Unilateral or bilateral, longitudinally extensive lesions involving the corticospinal tract occur commonly in Korean patients $^{8,9}$ and have occasionally been reported in other co- 

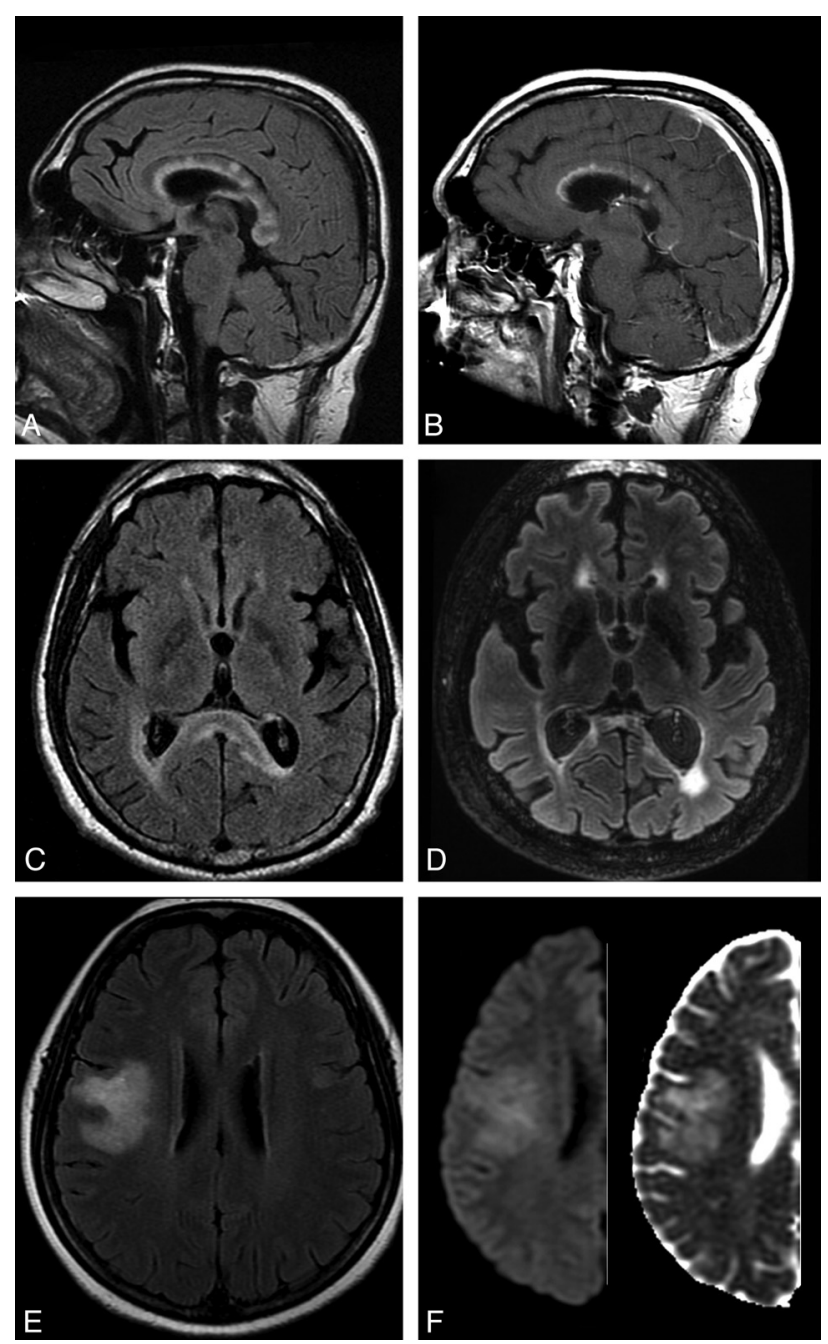

FIG 5. $A$ and C, FLAIR MR imaging shows acute, heterogeneous, "fluffy," corpus callosum lesions, with prominent splenial hyperintensity and swelling, during symptomatic cerebral AQP4-positive NMO relapse. $B$, Contrast-enhanced TIWI shows linear enhancement involving the undersurface of the corpus callosum in its anterior third and more focal enhancement within lesions in the body of the corpus callosum. D, 3T follow-up FLAIR MR imaging at 2.5 years shows severe splenial (and global cerebral) atrophy. E, FLAIR MR imaging shows a large, evanescent lesion in the right hemisphere in AQP4-negative NMO spectrum disorder. F, DWI (left) and ADC map (right) confirm facilitated diffusion in the lesion.

horts. $^{32,51}$ Such lesions may be associated with severe contralateral motor deficits.

\section{MR Imaging Enhancement Patterns}

While most reported NMO brain lesions do not show gadolinium enhancement, the frequency of acute lesional enhancement is unknown. Patchy enhancement with blurred margins ("cloudlike enhancement") in cerebral lesions has been reported as relatively specific for NMO (Fig 4A), 8,52 and the presence of asymptomatic "pencil-thin ependymal enhancement" (similar to that observed in infectious ependymitis) may serve to further differentiate the condition from MS (Fig 4C, -D). ${ }^{53,54}$ Rarely, NMO lesions exhibit a solid enhancement pattern that is indistinguishable from acute MS (Fig 4G, - H). Incomplete ring enhancement, a common feature in larger, acute MS lesions, has not, to the authors' knowledge, been described in NMO.

\section{ADVANCED MR IMAGING}

The recent application of advanced imaging techniques to NMO, including ultra-high-field MR imaging (7T), ${ }^{55}$ magnetization transfer (MT) imaging, ${ }^{56,57}$ double inversion recovery imaging, ${ }^{58}$ DTI, ${ }^{56,59-61}$ MR spectroscopy, ${ }^{57,62,63}$ iron-deposition imaging, ${ }^{64}$ and MR volumetric/morphometric assessments, ${ }^{56,58,65}$ is beginning to yield important insights into disease pathogenesis and to build on the MR imaging signature of the disease that has emerged from conventional imaging studies during the last 15 years. In addition, multiparametric approaches may provide complementary information in establishing nonconventional MR imaging patterns unique to patients with NMO (On-line Table).

\section{Cortical and Deep Gray Matter Pathology}

The presence of cortical lesions is almost universal in longstanding MS, and recent pathologic and imaging studies show that cortical pathology is common, even at first presentation. ${ }^{66}$ Cortical pathology correlates better with cognitive and motor parameters than white matter T2 lesion burden in MS, ${ }^{67}$ and gray matter atrophy has gained acceptance as an outcome measure in therapeutic clinical trials. In NMO, neuropathologic studies have thus far failed to demonstrate cortical pathology, ${ }^{68}$ though patient numbers studied are relatively low. Calabrese ${ }^{58}$ recently used double inversion recovery imaging, a technique that can reveal up to $18 \%$ (37\% with retrospective scoring) of the histopathologically confirmed cortical lesion load in MS, ${ }^{69}$ to study 30 patients with NMO, 30 with relapsing MS, and 30 healthy controls. While more than two-thirds of the patients with relapsing MS had cortical lesions, none were demonstrable in either patients with NMO or healthy controls. Mild regional reductions in cortical thickness, determined by using FreeSurfer (http://surfer.nmr. mgh.harvard.edu/) in the precentral, postcentral, and calcarine gyri, and a mild reduction in thalamic volume were observed in patients with NMO versus healthy controls. The authors attributed these changes to secondary axonal degeneration following severe spinal cord and anterior optic pathway inflammation. Using an alternative approach, Kister et $\mathrm{al}^{55}$ found no cortical lesions in 10 patients with NMO spectrum disorder studied with ultrahigh-field (7T); of 92 white matter lesions studied, only 8 were traversed by a central venule, a further distinction from MS that indicates a fundamental dichotomy in the pathogenesis of the 2 conditions.

Voxel-based morphometry has been used to compare gray matter structures in NMO, MS, and healthy controls. ${ }^{56,65,70}$ Duan et $\mathrm{al}^{65}$ documented regional gray matter atrophy in the frontal cortex, temporal cortex, right inferior parietal lobule, and right insula of patients with NMO $(n=26)$ versus controls, but these differences lost statistical significance when a family-wise error correction for multiple comparisons at the voxel level was applied. Conversely, marked atrophy of cortical and deep gray structures was present in patients with relapsing MS, and compared with NMO, significant gray matter volume loss was present in the thalami, caudate, mammillary body, parahippocampal gyrus, right hippocampus, and right insula. Chanson et $\mathrm{al}^{70}$ observed volume loss in the thalamus in patients with NMO $(n=30)$ versus healthy controls $(n=30)$, and Pichiecchio et $\mathrm{al}^{56}$ found a reduction in the attenuation 

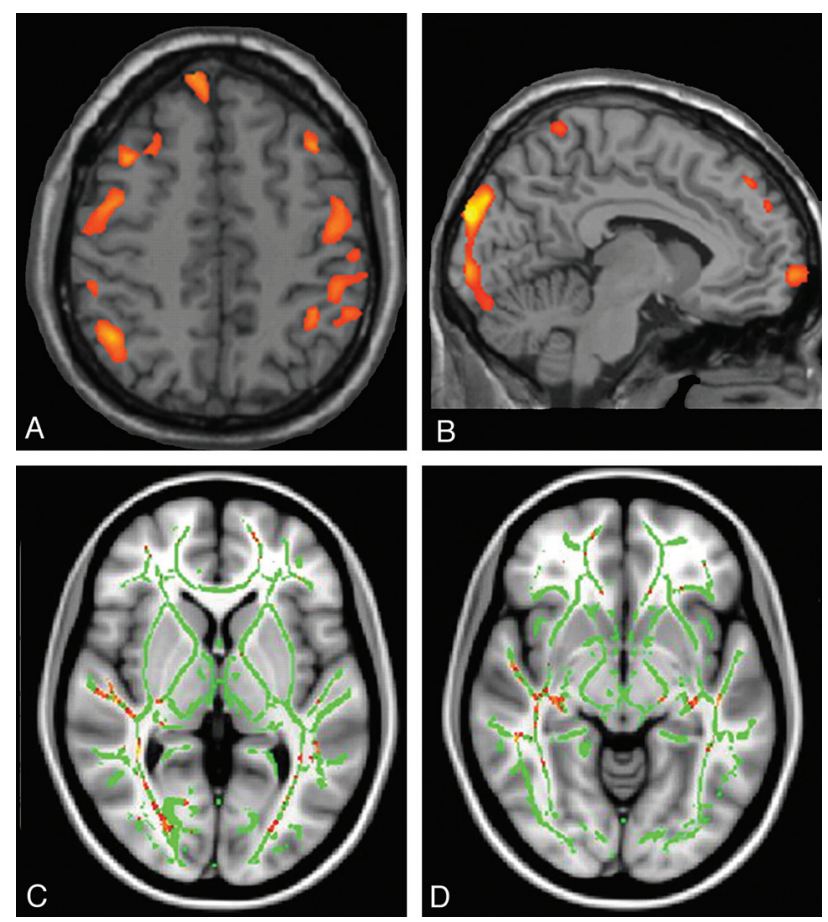

FIG 6. Voxel-based morphometry analysis shows a reduction in the attenuation and volume of gray matter in the motor $(A)$ and visual $(B)$ cortices in patients with NMO compared with the healthy controls. Voxel-based morphometry output is overlaid on the standard $\mathrm{CH}_{2}$ template. Voxelwise DTI analysis shows a significantly lowered fractional anisotropy along the pathway of the optic radiation bilaterally $(C)$ and involvement of both lateral geniculate nuclei $(D)$. Pichiecchio $A$, Tavazzi E, Poloni G, et al. Advanced magnetic resonance imaging of neuromyelitis optica: a multiparametric approach. Mult Scler 18(6):817-24, copyright ๑ 2012. Reprinted by Permission of SAGE.

and volume of gray matter in the motor and visual cortices and regions associated with executive and language functions in patients with NMO $(n=8)$ versus healthy controls $(n=7)$ by using a similar voxel-based morphometry method (Fig 6A, $-B$ ). MT imaging has been explored in NMO, thus far with discrepant results: Rocca et $\mathrm{al}^{57}$ observed a reduced MT ratio in normal-appearing gray matter in 10 patients with NMO, suggesting occult gray matter damage, while Pichiecchio et $\mathrm{al}^{56}$ observed no differences in either the cortical or deep gray matter MT ratio between patients $(n=8)$ and healthy controls $(n=7)$. Finally, while iron deposition in the deep gray matter structures is described in $\mathrm{MS}^{71}$ and may contribute to disease progression, ${ }^{72}$ Chen et $\mathrm{al}^{64}$ found no evidence of abnormal iron deposition in the putamen, globus pallidus, caudate nucleus, thalamus, substantia nigra, red nucleus, or dentate nucleus by using quantitative $3 \mathrm{D}$-enhanced susceptibilityweighted angiography in 42 patients with NMO compared with healthy controls.

In summary, nonconventional imaging data, while limited by small patient numbers and a variety of technical issues, indicate that the gray matter structures are less affected by NMO than by MS and, in particular, suggest that an absence of focal cortical lesions in NMO may differentiate the 2 conditions. In contradistinction to MS, the relative sparing of gray matter structures in NMO may explain the lack of a progressive phenotype in the condition.

\section{Normal-Appearing White Matter}

In patients with NMO and normal structural brain MR imaging findings, DTI has identified increased mean diffusivity and transverse $(\lambda 2,3)$ eigenvalues (reflecting the average diffusion coefficient perpendicular to the direction of maximal diffusivity) in the corticospinal tract and optic radiation, but not the cingulum or corpus callosum, ${ }^{60}$ implicating axonal degeneration secondary to spinal cord and anterior optic pathway lesions. Liu et $\mathrm{al}^{59}$ inferred the presence of subtle, widespread pathology in NMO normalappearing white matter from an analysis of white matter connectivity matrices derived from deterministic tractography performed in 26 patients with NMO. In a more recent DTI study, Zhao et $\mathrm{al}^{61}$ confirmed the presence of occult damage to normalappearing white matter in relapsing NMO but found that while mean diffusivity was increased in regions of interest in the optic radiation (and genu of the corpus callosum) versus controls, reduced values were observed in the internal capsule, thalamus, and medulla oblongata. Furthermore, Pichiecchio et $\mathrm{al}^{56}$ examined mean diffusivity histogram-derived metrics of normal-appearing white matter in NMO and healthy controls and found no significant differences, while tract-based spatial statistics in the same cohort identified lowered fractional anisotropy in the optic radiation bilaterally (Fig $6 C,-D)$.

Quantitative structural brain assessment using SIENAX software (http://fsl.fmrib.ox.ac.uk/fsl/fslwiki/SIENA) has demonstrated a significant reduction in total white matter volume in patients with NMO $(n=30)$, even in the absence of T2 hyperintensities, versus healthy controls $(n=30) .{ }^{70}$ Regional voxelbased morphometry analysis in the same cohort identified possible volume loss clustered in the optic radiations and corpus callosum.

Finally, the metabolic pattern of normal-appearing white matter in NMO/NMO spectrum disorder, as assessed by multivoxel chemical shift imaging at $1.5 \mathrm{~T}^{56,63}$ and $3 \mathrm{~T}^{62}$ with ${ }^{1} \mathrm{H}-\mathrm{MR}$ spectroscopy, is identical to that in controls.

Taken together, these studies indicate subtle and, with the possible exception of the corpus callosum, selective structural alterations in the optic and motor pathways in the brain, implicating trans-synaptic axonal degeneration secondary to destructive lesions in the optic nerves and spinal cord, respectively. The normal spectroscopy data suggest that the diffuse degenerative process that affects the white matter in MS is lacking in NMO. However, variability in the severity of the disease and the exclusion of cases with brain abnormalities may have confounded the results of these studies, and larger multiparametric studies of well-phenotyped patients are required.

\section{Spinal Cord}

Few studies have assessed the application of nonconventional MR imaging techniques to the spinal cord in NMO. Filippi et $\mathrm{al}^{17}$ were unable to demonstrate a difference in cervical cord MT ratio histogram metrics in clinically defined NMO $(n=8)$ versus MS ( $n=$ 10 ), despite cord T1 hypointensity and atrophy being restricted to the NMO cases. Two studies have used DTI in the assessment of cervical spinal cord damage in NMO. In the first, Qian et $\mathrm{al}^{73}$ showed abnormal DTI-derived metrics (especially fractional anisotropy) in the cervical spinal cord of patients with $\mathrm{NMO}(n=$ 
$10)$, even in the absence of signal alteration on conventional T2WI. Similarly, there was no correlation between diffusivity alterations and the number or extent of focal $\mathrm{T} 2$ hyperintensities in the cervical cord of patients with MS. ${ }^{74}$ In the second, Klawiter et $\mathrm{al}^{75}$ demonstrated higher radial diffusivity within damaged (as assessed by T2WI) white matter tracts in NMO $(n=10)$ versus MS $(n=10)$, consistent with the more destructive nature of the former condition. DTI metrics of damaged gray matter regions did not differentiate NMO and MS. These studies indicate that DTI may inform the pathology of spinal cord disease in NMO. Specific measures such as fractional anisotropy may provide quantitative biomarkers of white matter damage not readily identified by conventional MR imaging techniques.

\section{CONCLUSIONS}

The seminal discovery of serum AQP4 antibodies in patients with NMO has validated earlier clinical and radiologic observations that separated the condition from conventional MS. While the presence of LESCLs suggests NMO and expedites referral for appropriate serologic testing, the absence of serum AQP4 antibodies in patients with classic clinical and radiologic features and their presence in patients with a spectrum of brain lesions have recently led to a re-appraisal of current diagnostic criteria for the disease. Recommendations by an international task force ${ }^{76}$ to include newly recognized imaging features of NMO, such as periependymal brain lesions, emphasize the importance of MR imaging in the diagnostic work-up of patients with CNS inflammatory disease.

Greater scrutiny of brain MR imaging in patients with NMO has revealed an expanding array of lesion subtypes with which the neuroradiologist must become familiar. The integration of imaging with clinical and laboratory data in individual patients facilitates rapid differentiation of NMO/NMO spectrum disorder from MS. This distinction has critical therapeutic implications: NMO does not respond to traditional MS immunomodulatory therapy with $\beta$-interferon, which in some patients may even be deleterious. ${ }^{13,14}$ Similarly, severe exacerbations of NMO have been reported in patients treated with natalizumab, ${ }^{5}$ an efficacious monoclonal antibody therapy for MS. Case series do suggest that NMO responds to immunosuppressive therapy and, in particular, to the B-cell-depleting anti-CD20 monoclonal antibody, rituximab. ${ }^{77}$ Of relevance to the neuroradiologist, rituximab has been associated with progressive multifocal leukoencephalopathy in patients with systemic autoimmune disease, ${ }^{78}$ and heightened vigilance for this complication in patients with $\mathrm{NMO}$ is paramount.

Nonconventional brain imaging is beginning to offer new insights into the pathophysiology of NMO and further differentiates the condition from MS. The lack of focal cortical lesions as assessed by ultra-high-field MR imaging and double inversion recovery imaging is of particular interest; if confirmed, double inversion recovery sequences may be helpful in distinguishing NMO from MS in difficult clinical cases. Early DTI, MT imaging, and MR spectroscopy data indicate an absence of diffuse "degenerative" disease in the normal-appearing white and gray matter in $\mathrm{NMO}$, consistent with the lack of a progressive phenotype in this condition, an important distinction from MS that may also have therapeutic repercussions.

Conventional and nonconventional neuroimaging investigations in NMO have accelerated in tandem with rapid developments in the understanding of NMO pathobiology during the past 5-10 years. These studies underscore the importance of neuroimaging in the diagnosis and monitoring of NMO and emphasize its role in directing the early commencement of targeted immunotherapy.

\section{ACKNOWLEDGMENTS}

The authors are grateful to Professor Jun-ichi Kira and Dr Koji Shinoda for kindly providing the images included in Fig 3.

Disclosures: Michael H. Barnett—UNRELATED: Board Membership: Biogen Idec, Novartis, Bayer, Genzyme, Comments: Scientific advisory boards, Grants/Grants Pending: Novartis, ${ }^{*}$ Payment for Lectures (including service on Speakers Bureaus): Novartis, Biogen Idec, Sanofi-Aventis, Genzyme, Comments: Independent scientific presentations, Travel/Accommodations/Meeting Expenses Unrelated to Activities Listed: Novartis. Yael Barnett—UNRELATED: Grants/Grants Pending: Biogen Idec.* Mahtab Ghadiri-UNRELATED: Grants/Grants Pending: Novartis,* Payment for Lectures (including service on Speakers Bureaus): Bayer, Travel/Accommodations/ Meeting Expenses Unrelated to Activities Listed: Novartis, CSL, Genzyme. Lynette Masters-UNRELATED: Payment for Lectures (including service on Speakers Bureaus): Biogen Idec, Novartis, Comments: independent scientific presentation. Robert Zivadinov—UNRELATED: Consultancy: Biogen Idec, EMD Serono, Teva Pharmaceuticals, Genzyme-Sanofi, Novartis, Grants/Grants Pending: Biogen Idec, EMD Serono, Teva Pharmaceuticals, Novartis, Genzyme-Sanof, Payment for Lectures (including service on Speakers Bureaus): Biogen Idec, EMD Serono, Teva Pharmaceuticals, Genzyme-Sanofi, Novartis. *Money paid to the institution.

\section{REFERENCES}

1. Devic E. Myélite subaiguë compliquée de névrite optique. Bull Med (Paris) 1894;8:1033-34

2. Wingerchuk DM, Hogancamp WF, O'Brien PC, et al. The clinical course of neuromyelitis optica (Devic's syndrome). Neurology 1999;53:1107-14

3. Lennon VA, Wingerchuk DM, Kryzer TJ, et al. A serum autoantibody marker of neuromyelitis optica: distinction from multiple sclerosis. Lancet 2004;364:2106-12

4. Lennon VA, Kryzer TJ, Pittock SJ, et al. IgG marker of optic-spinal multiple sclerosis binds to the aquaporin- 4 water channel. $J$ Exp Med 2005;202:473-77

5. Barnett MH, Prineas JW, Buckland ME, et al. Massive astrocyte destruction in neuromyelitis optica despite natalizumab therapy. Mult Scler 2012;18:108-12

6. Lucchinetti CF, Mandler RN, McGavern D, et al. A role for humoral mechanisms in the pathogenesis of Devic's neuromyelitis optica. Brain 2002;125:1450-61

7. Giovannoni G. To test or not to test: NMO-IgG and optic neuritis. Neurology 2008;70:2192-93

8. Kim W, Park MS, Lee SH, et al. Characteristic brain magnetic resonance imaging abnormalities in central nervous system aquaporin-4 autoimmunity. Mult Scler 2010;16:1229-36

9. Kim W, Kim SH, Huh SY, et al. Brain abnormalities in neuromyelitis optica spectrum disorder. Mult Scler Int 2012;2012:735486

10. Matiello M, Lennon VA, Jacob A, et al. NMO-IgG predicts the outcome of recurrent optic neuritis. Neurology 2008;70:2197-200

11. Weinshenker BG, Wingerchuk DM, Vukusic S, et al. Neuromyelitis optica IgG predicts relapse after longitudinally extensive transverse myelitis. Ann Neurol 2006;59:566-69

12. Wingerchuk DM, Lennon VA, Pittock SJ, et al. Revised diagnostic criteria for neuromyelitis optica. Neurology 2006;66:1485-89

13. Palace J, Leite MI, Nairne A, et al. Interferon beta treatment in neuromyelitis optica: increase in relapses and aquaporin 4 antibody titers. Arch Neurol 2010;67:1016-17

14. Shimizu J, Hatanaka Y, Hasegawa M, et al. IFN $\boldsymbol{\beta}$-1b may severely 
exacerbate Japanese optic-spinal MS in neuromyelitis optica spectrum. Neurology 2010;75:1423-27

15. Min JH, Kim BJ, Lee KH. Development of extensive brain lesions following fingolimod (FTY720) treatment in a patient with neuromyelitis optica spectrum disorder. Mult Scler 2012;18:113-15

16. Nakamura M, Miyazawa I, Fujihara K, et al. Preferential spinal central gray matter involvement in neuromyelitis optica: an MRI study. J Neurol 2008;255:163-70

17. Filippi M, Rocca MA, Moiola L, et al. MRI and magnetization transfer imaging changes in the brain and cervical cord of patients with Devic's neuromyelitis optica. Neurology 1999;53:1705-10

18. Filippi M, Rocca MA. MR imaging of Devic's neuromyelitis optica. Neurol Sci 2004;25(suppl 4):S371-73

19. Pires CE, Silva CM, Lopes FC, et al. Brain MRI abnormalities in Brazilian patients with neuromyelitis optica. J Clin Neurosci 2012;19:969-74

20. Downer JJ, Leite MI, Carter R, et al. Diagnosis of neuromyelitis optica (NMO) spectrum disorders: is MRI obsolete? Neuroradiology 2012;54:279-85

21. Hinson SR, Romero MF, Popescu BF, et al. Molecular outcomes of neuromyelitis optica (NMO)-IgG binding to aquaporin-4 in astrocytes. Proc Natl Acad Sci U S A 2012;109:1245-50

22. Magaña SM, Matiello M, Pittock SJ, et al. Posterior reversible encephalopathy syndrome in neuromyelitis optica spectrum disorders. Neurology 2009;72:712-17

23. Delalande S, de Seze J, Fauchais AL, et al. Neurologic manifestations in primary Sjögren syndrome: a study of 82 patients. Medicine (Baltimore) 2004;83:280-91

24. Schulz SW, Shenin M, Mehta A, et al. Initial presentation of acute transverse myelitis in systemic lupus erythematosus: demographics, diagnosis, management and comparison to idiopathic cases. Rheumatol Int 2012;32:2623-27

25. McKeon A, Lennon VA, Jacob A, et al. Coexistence of myasthenia gravis and serological markers of neurological autoimmunity in neuromyelitis optica. Muscle Nerve 2009;39:87-90

26. Pittock SJ, Lennon VA, de SJ, et al. Neuromyelitis optica and non organ-specific autoimmunity. Arch Neurol 2008;65:78-83

27. Kim SM, Waters P, Vincent A, et al. Sjögren's syndrome myelopathy: spinal cord involvement in Sjögren's syndrome might be a manifestation of neuromyelitis optica. Mult Scler 2009;15:1062-68

28. Trebst C, Raab P, Voss EV, et al. Longitudinal extensive transverse myelitis: it's not all neuromyelitis optica. Nat Rev Neurol 2011;7:688-98

29. Khanna S, Sharma A, Huecker J, et al. Magnetic resonance imaging of optic neuritis in patients with neuromyelitis optica versus multiple sclerosis. J Neuroophthalmol 2012;32:216-20

30. Li Y, Xie P, Lv F, et al. Brain magnetic resonance imaging abnormalities in neuromyelitis optica. Acta Neurol Scand 2008;118:218-25

31. McKeon A, Lennon VA, Lotze T, et al. CNS aquaporin-4 autoimmunity in children. Neurology 2008;71:93-100

32. Pittock SJ, Lennon VA, Krecke K, et al. Brain abnormalities in neuromyelitis optica. Arch Neurol 2006;63:390-96

33. Cabrera-Gómez JA, Quevedo-Sotolongo L, Gonzalez-Quevedo A, et al. Brain magnetic resonance imaging findings in relapsing neuromyelitis optica. Mult Scler 2007;13:186-92

34. Makino T, Ito S, Mori M, et al. Diffuse and heterogeneous T2-hyperintense lesions in the splenium are characteristic of neuromyelitis optica. Mult Scler 2013;19:308-15

35. Pittock SJ, Weinshenker BG, Lucchinetti CF, et al. Neuromyelitis optica brain lesions localized at sites of high aquaporin 4 expression. Arch Neurol 2006;63:964-68

36. Poppe AY, Lapierre Y, Melancon D, et al. Neuromyelitis optica with hypothalamic involvement. Mult Scler 2005;11:617-21

37. Parratt JD, Prineas JW. Neuromyelitis optica: a demyelinating disease characterized by acute destruction and regeneration of perivascular astrocytes. Mult Scler 2010;16:1156-72
38. Wang F, Liu Y, Duan Y, et al. Brain MRI abnormalities in neuromyelitis optica. Eur J Radiol 2011;80:445-49

39. Barkhof F, Filippi M, Miller DH, et al. Comparison of MRI criteria at first presentation to predict conversion to clinically definite multiple sclerosis. Brain 1997;120(pt 11):2059-69

40. Polman $\mathrm{CH}$, Reingold SC, Banwell B, et al. Diagnostic criteria for multiple sclerosis: 2010 revisions to the McDonald criteria. Ann Neurol 2011;69:292-302

41. Nakashima I, Fujihara K, Miyazawa I, et al. Clinical and MRI features of Japanese patients with multiple sclerosis positive for NMO-IgG. J Neurol Neurosurg Psychiatry 2006;77:1073-75

42. Viegas S, Weir A, Esiri M, et al. Symptomatic, radiological and pathological involvement of the hypothalamus in neuromyelitis optica. J Neurol Neurosurg Psychiatry 2009;80:679-82

43. Nakajima H, Fujiki Y, Ito T, et al. Anti-aquaporin-4 antibody-positive neuromyelitis optica presenting with syndrome of inappropriate antidiuretic hormone secretion as an initial manifestation. Case Rep Neurol 2011;3:263-67

44. Baba T, Nakashima I, Kanbayashi T, et al. Narcolepsy as an initial manifestation of neuromyelitis optica with anti-aquaporin-4 antibody. J Neurol 2009;256:287-88

45. Suzuki K, Nakamura T, Hashimoto K, et al. Hypothermia, hypotension, hypersomnia, and obesity associated with hypothalamic lesions in a patient positive for the anti-aquaporin 4 antibody: a case report and literature review. Arch Neurol 2012;69:1355-59

46. Lu Z, Qiu W, Zou Y, et al. Characteristic linear lesions and longitudinally extensive spinal cord lesions in Chinese patients with neuromyelitis optica. J Neurol Sci 2010;293:92-96

47. Nakamura M, Misu T, Fujihara K, et al. Occurrence of acute large and edematous callosal lesions in neuromyelitis optica. Mult Scler 2009; 15:695-700

48. Cabrera-Gomez JA, Kister I. Conventional brain MRI in neuromyelitis optica. Eur J Neurol 2012;19:812-19

49. Saiki S, Ueno Y, Moritani T, et al. Extensive hemispheric lesions with radiological evidence of blood-brain barrier integrity in a patient with neuromyelitis optica. J Neurol Sci 2009;284:217-19

50. Barnett MH, Sutton I. Neuromyelitis optica: not a multiple sclerosis variant. Curr Opin Neurol 2012;25:215-20

51. Bichuetti DB, Oliveira EM, Souza NA, et al. Neuromyelitis optica in Brazil: a study on clinical and prognostic factors. Mult Scler 2009;15:613-19

52. Ito S, Mori M, Makino T, et al. "Cloud-like enhancement" is a magnetic resonance imaging abnormality specific to neuromyelitis optica. Ann Neurol 2009;66:425-28

53. Banker P, Sonni S, Kister I, et al. Pencil-thin ependymal enhancement in neuromyelitis optica spectrum disorders. Mult Scler 2012;18:1050-53

54. McGraw CA, Swerdlow ML, Robbins MS. Acute reversible periependymal ventricular enhancement in neuromyelitis optica. Eur J Neurol 2012;19:e57-e58

55. Kister I, Herbert J, Zhou Y, et al. Ultrahigh-field MR (7T) imaging of brain lesions in neuromyelitis optica. Mult Scler Int 2013;2013:398259

56. Pichiecchio A, Tavazzi E, Poloni G, et al. Advanced magnetic resonance imaging of neuromyelitis optica: a multiparametric approach. Mult Scler 2012;18:817-24

57. Rocca MA, Agosta F, Mezzapesa DM, et al. Magnetization transfer and diffusion tensor MRI show gray matter damage in neuromyelitis optica. Neurology 2004;62:476-78

58. Calabrese M, Oh MS, Favaretto A, et al. No MRI evidence of cortical lesions in neuromyelitis optica. Neurology 2012;79:1671-76

59. Liu Y, Duan Y, He Y, et al. Altered topological organization of white matter structural networks in patients with neuromyelitis optica. PLoS One 2012;7:e48846

60. Yu C, Lin F, Li K, et al. Pathogenesis of normal-appearing white matter damage in neuromyelitis optica: diffusion-tensor MR imaging. Radiology 2008;246:222-28

61. Zhao DD, Zhou HY, Wu QZ, et al. Diffusion tensor imaging char- 
acterization of occult brain damage in relapsing neuromyelitis optica using 3.0T magnetic resonance imaging techniques. Neuroimage 2012;59:3173-77

62. Aboul-Enein F, Krssak M, Hoftberger R, et al. Diffuse white matter damage is absent in neuromyelitis optica. AJNR Am J Neuroradiol 2010;31:76-79

63. de Seze J, Blanc F, Kremer S, et al. Magnetic resonance spectroscopy evaluation in patients with neuromyelitis optica. J Neurol Neurosurg Psychiatry 2010;81:409-11

64. Chen X, Zeng C, Luo T, et al. Iron deposition of the deep grey matter in patients with multiple sclerosis and neuromyelitis optica: a control quantitative study by $3 \mathrm{D}$-enhanced susceptibility-weighted angiography (ESWAN). Eur J Radiol 2012;81:e633-e639

65. Duan Y, Liu Y, Liang P, et al. Comparison of grey matter atrophy between patients with neuromyelitis optica and multiple sclerosis: a voxel-based morphometry study. Eur J Radiol 2012;81:e110-e114

66. Lucchinetti CF, Popescu BF, Bunyan RF, et al. Inflammatory cortical demyelination in early multiple sclerosis. $N$ Engl $J$ Med 2011;365:2188-97

67. Calabrese M, Rinaldi F, Grossi P, et al. Cortical pathology and cognitive impairment in multiple sclerosis. Expert Rev Neurother 2011;11:425-32

68. Popescu BF, Parisi JE, Cabrera-Gomez JA, et al. Absence of cortical demyelination in neuromyelitis optica. Neurology 2010;75:2103-09

69. Seewann A, Kooi EJ, Roosendaal SD, et al. Postmortem verification of MS cortical lesion detection with 3D DIR. Neurology 2012; 78:302-08

70. Chanson JB, Lamy J, Rousseau F, et al. White matter volume is de- creased in the brain of patients with neuromyelitis optica. Eur J Neurol 2013;20:361-67

71. Habib CA, Liu M, Bawany N, et al. Assessing abnormal iron content in the deep gray matter of patients with multiple sclerosis versus healthy controls. AJNR Am J Neuroradiol 2012;33:252-58

72. Zivadinov R, Heininen-Brown M, Schirda CV, et al. Abnormal subcortical deep-gray matter susceptibility-weighted imaging filtered phase measurements in patients with multiple sclerosis: a case-control study. Neuroimage 2012;59:331-39

73. Qian W, Chan Q, Mak H, et al. Quantitative assessment of the cervical spinal cord damage in neuromyelitis optica using diffusion tensor imaging at 3 Tesla. J Magn Reson Imaging 2011;33:1312-20

74. Agosta F, Absinta M, Sormani MP, et al. In vivo assessment of cervical cord damage in MS patients: a longitudinal diffusion tensor MRI study. Brain 2007;130:2211-19

75. Klawiter EC, Xu J, Naismith RT, et al. Increased radial diffusivity in spinal cord lesions in neuromyelitis optica compared with multiple sclerosis. Mult Scler 2012;18:1259-68

76. Miller DH, Weinshenker BG, Filippi M, et al. Differential diagnosis of suspected multiple sclerosis: a consensus approach. Mult Scler 2008;14:1157-74

77. Bedi GS, Brown AD, Delgado SR, et al. Impact of rituximab on relapse rate and disability in neuromyelitis optica. Mult Scler 2011;17:1225-30

78. Carson KR, Evens AM, Richey EA, et al. Progressive multifocal leukoencephalopathy after rituximab therapy in HIV-negative patients: a report of $\mathbf{5 7}$ cases from the Research on Adverse Drug Events and Reports project. Blood 2009;113:4834-40 